\title{
Virtual Reality for Dose Optimization in Pediatric Nuclear Medicine: Better Than the Real Thing
}

$\mathbf{T}$ he use of nuclear medicine imaging and $\mathrm{CT}$ has grown dramatically over the last several decades. In the Unites States, the annual number of nuclear medicine procedures increased 3 -fold (from 7 to 20 million) and the annual number of CT procedures 20fold (from 3 to 60 million) between 1985 and 2005 (1). As a result of this increased medical exposure of the population, the average (i.e., per-capita) annual background dose in the Unites States has nearly doubled, from 3.0 to $5.6 \mathrm{mSv}$ (2). There has been increasing societal concern over the potential public health impactnamely, an increased risk of cancerassociated with this dramatic increase in exposure from diagnostic imaging.

See page 1923

Brenner and Hall, for example, have estimated that as much as $2 \%$ of all cancers in the United States may be attributable to CT irradiation (3). Although nuclear medicine diagnostic procedures are not performed as frequently as CT scans and the organ absorbed doses are generally not as high, the effective dose, and therefore the overall radiogenic cancer risk per procedure, are comparable (4). Radiogenic cancer risks vary with sex and, in particular, age at exposure, with children at markedly greater risk than adults. For example, the lifetime

Received Oct. 24, 2011; revision accepted Oct. 26, 2011.

For correspondence or reprints contact: Pat Zanzonico, Department of Medical Physics, 1250 York Ave., New York, NY 10021.

E-mail: zanzonip@mskcc.org

Published online Nov. 11, 2011.

COPYRIGHT @ 2011 by the Society of Nuclear Medicine, Inc.

DOI: 10.2967/jnumed.111.097188 attributable risk (LAR) of a radiationinduced cancer is approximately $20 \% /$ $\mathrm{Sv}$ at $10 \mathrm{y}$ of age but only about $7 \% / \mathrm{Sv}$ at $40 \mathrm{y}$ of age (5). Therefore, the management of radiation doses associated with diagnostic imaging in pediatric patients is a particular and persisting concern.

As illustrated in Figure 1, for any radiologic procedure, the image quality - the aesthetics of the study, if you will-improves progressively with patient dose (6). In the case of nuclear medicine, for example, as the administered activity is increased and more photons emanate from the patient and strike the imaging detector, the quantum mottle in the resulting image is reduced, and the image appears smoother and more visually aesthetic. More importantly, diagnostic information content (e.g., lesion detectability) increases as well with increasing dose-but only to a certain point. Although image aesthetics will continue to improve with increasing dose, the optimum patient dose is reached once all of the diagnostic information technically derivable from a particular study has been obtained. Increasing the administered activity and therefore the dose further yields no additional information and thus unnecessarily increases the radiogenic cancer risk to the patient. The optimum-dose concept is the basis of minimizing the risk associated with diagnostic imaging and of the Image Gently campaign currently being promoted by many agencies and professional organizations (7-10). For all diagnostic procedures, the study parameters should be judiciously selected to deliver the minimum radiation dose consistent with yielding the clinical information being sought. The current computer simulation study by Sgouros et al.
(11) is a rigorously elegant case study of how this may be accomplished in the context of nuclear medicine.

In this study, pharmacokinetic modeling and state-of-the-art pediatric nonuniform rational B-spline (NURBS)-based anatomic phantoms of 10-y-old girls were used to simulate SPECT images of the renal agent ${ }^{99 \mathrm{~m}}$ Tc-dimercaptosuccinic acid (DMSA). Importantly, 2 different anatomic phantoms of 10 -y-old girls were considered; the 2 phantoms had the same body mass $(32 \mathrm{~kg}$ ) but different body morphometry, short and stout $(125 \mathrm{~cm}$ tall) versus tall and lean (147 cm tall). The fidelity of this analysis to actual clinical imaging is remarkable and distinguishes it from other such analyses. Different administered activities $(25 \%, 50 \%, 75 \%$, $100 \%, 125 \%$, and $150 \%$ of the standard mass-based administered activity of $1.85 \mathrm{MBq} / \mathrm{kg}$ ), 2.2-cm lesions with different target-to-background activity concentration ratios and at different intrarenal locations, and 50 random variations of lesion uptake for each location were considered. The simulated projection image data were scaled to yield the counts that would be obtained using a dual-detector $\gamma$-camera system with low-energy high-resolution collimation (system sensitivity, $85.6 \mathrm{cps/}$ $\mathrm{MBq}$ of ${ }^{99 \mathrm{~m}} \mathrm{Tc}$ ) and a 30-min acquisition and with incorporation of the effects of scatter, attenuation, and detector response. The NURBS phantoms were also used to calculate ${ }^{99 \mathrm{~m}} \mathrm{Tc} \mathrm{S}$ factors by separate energy spectrum-weighted Monte Carlo simulations for the particulate and photon radiations. Channelized Hotelling observer methodology was used in a receiver-operating-characteristic analysis of lesion detectabilityin the simulated reconstructed images. The 


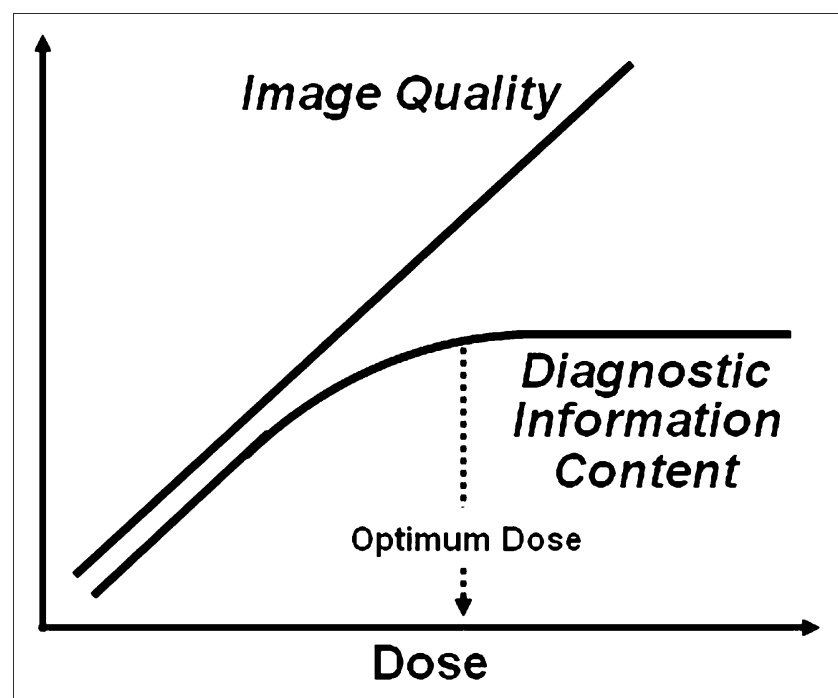

FIGURE 1. Optimum-dose concept. Stylized graft illustrating relationship among image quality, diagnostic information content, and patient dose for diagnostic imaging procedures. (Reprinted with permission of (6).)

MIRD Committee formalism and the foregoing ${ }^{99 \mathrm{~m} T c-D M S A}$ pharmacokinetics and ${ }^{99 \mathrm{~m}} \mathrm{Tc} \mathrm{S}$ factors were used to calculate the mean organ-absorbed doses to the 2 pediatric phantoms. The absorbed doses thus calculated ranged from $3.9 \times 10^{-5}$ (salivary glands) to $7.4 \times 10^{-2}$ (liver) $\mathrm{mGy} / \mathrm{MBq}$ in the short, stout phantom and $1.1 \times 10^{-4}$ (salivary glands) to $5.4 \times 10^{-2}$ (liver) $\mathrm{mGy} / \mathrm{MBq}$ in the tall, lean phantom. Organ-absorbed doses were then converted to LARs of cancer using BEIR VII age- and sex-specific risk factors (5).

Importantly, the same diagnostic performance-in terms of identifying the intrarenal defects - could be achieved in the tall, thin patient with only onehalf of the standard mass-based administered activity $(\mathrm{MBq} / \mathrm{kg})$ used for the short, stout patient. The difference in diagnostic information content between the 2 image sets was due to a combination of $10 \%$ more photons escaping, a $10 \%$ smaller average radius of the $\gamma$-camera orbit, and a $10 \%$ lower scatter-to-primary photon ratio for the tall, lean patient than for the short, stout patient. This combination resulted in slightly lower quantum mottle (noise), sharper spatial resolution, and higher contrast, respectively — and better diagnostic performance per unit adminis- tered activity-for the taller patient. For ${ }^{99 \mathrm{~m} T c-D M S A}$ renal imaging of children, therefore, the optimum administered activity $(\mathrm{MBq} / \mathrm{kg})$ and resulting radiation doses (mSv/MBq) are considerably lower for tall, lean patients than for short, stout patients of the same body mass. Specifically, the diagnostic information content of ${ }^{99 \mathrm{~m} T c-D M S A}$ renal imaging was optimized with an administered activity of $59 \mathrm{MBq}$ in a short, stout 10 -y-old girl but with only $30 \mathrm{MBq}$ in a tall, lean 10-y-old girl, resulting in a LAR of cancer of $0.006 \%$ for the short, stout patient but only $0.002 \%$ for the tall, lean patient. Similar LARs were obtained on the basis of the effective dose and the overall radiation detriment factor in ICRP 103 (12). First-order metrics of body morphometry such as body mass and body surface area are thus inadequate for optimum scaling of administered activities among patients.

The diagnostic information content of a procedure must, of course, not be compromised in a misguided attempt to minimize patient radiation dose. A suboptimal procedure (e.g., with an inadequate administered activity) not only compromises patient management by failing to disclose the information being sought but also may ultimately increase the patient's radiation exposure by requiring a repeated, properly performed study to obtain the needed information. Given the possibility of performing such suboptimal procedures in the interest of dose reduction, rigorous validation of diagnostic protocols designed to reduce patient exposure are therefore required before they are recommended for standard practice. In the current virtual-reality study by Sgouros et al. (11), such validation was accomplished by remarkably realistic computer simulation of SPECT studies over a range of administered activities in combination with comparison of diagnostic information content of the resulting images using receiver operating characteristics. Alternatively, such an analysis could be performed empirically - that is, in the context of a traditional clinical trial in which different patient strata are administered different activities of a radiopharmaceutical and the diagnostic accuracy correlated with the administered activity. In addition to practical limitations such as time and cost, patient-to-patient variations in the location and extent of defects and body morphometry and the absence of an unambiguous reference standard (i.e., ground truth diagnosis) may confound interpretation of the results and thus derivation of an optimum administered activity. Further, ethical considerations, especially in children, may make such a study unacceptable to institutional reviews boards and prospective subjects or their parents.

With persisting concerns regarding radiation risks of diagnostic imaging procedures, the virtual-reality approach of Sgouros et al. (11) can yield computationally validated "isoradiation risk"administered activity guidelines for pediatric and other patients that account for differences in body morphometry and body mass among patients - all while circumventing the burdens and limitations of traditional clinical trials. With the rigor achieved in the current study, virtual reality is indeed better than the real thing.

\section{Pat Zanzonico}

Memorial Sloan-Kettering Cancer Center New York, New York 


\section{REFERENCES}

1. Amis ES Jr, Butler PF, Applegate KE, et al. American College of Radiology white paper on radiation dose in medicine. J Am Coll Radiol. 2007;4:272-284.

2. Mettler FA Jr, Bhargavan M, Faulkner K, et al. Radiologic and nuclear medicine studies in the United States and worldwide: frequency, radiation dose, and comparison with other radiation sources: 1950-2007. Radiology. 2009;253:520-531.

3. Brenner DJ, Hall EJ. Computed tomography: an increasing source of radiation exposure. $N$ Engl J Med. 2007;357:2277-2284.

4. Mettler FA Jr, Huda W, Yoshizumi TT, Mahesh M. Effective doses in radiology and diagnostic nuclear medicine: a catalog. Radiology. 2008;248:254-263.
5. National Research Council, Committee on the Biological Effects of Ionizing Radiation. (BEIR VII-Phase 2), Health Risks From Exposure to Low Levels of Ionizing Radiation. Washington, DC: National Academy Press; 2006.

6. Zanzonico P. Dose optimization in coronary CTA. JACC Cardiovasc Imaging. 2010;3:1124-1126.

7. Goske MJ, Applegate KE, Boylan J, et al. Image GentlySM: a national education and communication campaign in radiology using the science of social marketing. J Am Coll Radiol. 2008;5:1200-1205.

8. Goske MJ, Applegate KE, Boylan J, et al. The Image Gently campaign: working together to change practice. AJR. 2008;190:273-274.

9. Goske MJ, Frush DP, Schauer DA. Image Gently campaign promotes radiation protection for children. Radiat Prot Dosimetry. 2009;135:276.
10. Goske MJ, Applegate $\mathrm{KE}$, Bell C, et al. Image Gently: providing practical educational tools and advocacy to accelerate radiation protection for children worldwide. Semin Ultrasound CT MR. 2010;31:57-63.

11. Sgouros G, Frey EC, Bolch WE, Wayson MB, Abadia AS, Treves ST. An approach for balancing diagnostic image quality with cancer risk: application to pediatric diagnostic imaging of ${ }^{99 m}$ Tc-dimercaptosuccinic acid. J Nucl Med. 2011;52:1923-1929.

12. International Commission on Radiological Protection (ICRP). ICRP publication 103: recommendations of the International Commission on Radiological Protection. Ann ICRP. 2007;37:1332 . 